Allen, R.B., and Nalluru, S., Exploring History with Narrative Timelines. HCII,

2009, LNCS 5617, pp 333-338, 2009.

\title{
Exploring History with Narrative Timelines
}

\author{
Robert B. Allen and Sumanth Nalluru \\ College of Information Science and Technology, Drexel University, \\ Philadelphia PA, 19104, USA \\ \{rba, sumanth\}@drexel.edu
}

\begin{abstract}
We develop novel timeline interfaces which separate the events in timelines into threads and then allow users to select among them. This interface is illustrated with five threads describing the causes of the American Civil War. In addition to selecting each of the threads, the sequence of events it describes can be played. That is, the user can step through the sequence of events and get a description of each event in the context of its thread. In addition, many of the events have links to more focused timelines and to external resources such as Wikipedia.
\end{abstract}

Keywords: Causation, Events, History, Interactivity, Narrative, Timelines, Visualization

\section{Introduction}

Timelines are a common graphical technique which present ordered sets of events. But, the events are often jumbled with several different threads interlaced. For instance, a timeline of the events leading to the American Civil War might include constitutional debates, Abolitionist protests, and changing agricultural patterns. While simple timelines help people to understand the temporal relationships among events, we believe that disentangling the threads among those events would make the entire sets of events more comprehensible. Indeed, visualization could enhance texts and be a sort of cognitive organizer.

This paper extends a series of interface techniques for interaction with timelines. Allen [1] described several ways timelines can be interactive such as by filtering the displayed items by attributes. Allen [2] introduced focus-context timelines which have two segments. One of these presented focus events and the other displays context events. That interface provided menus to the user for controlling specifically which sets of events are presented. The Lifelines project [3] developed a hierarchical timeline viewer for presenting significant life events such as the duration of medical conditions and treatments in patients.

Other interfaces have described techniques for presenting narrative and causal threads. For instance, Allen and Acheson (e.g., [4]) describe narrative lines which are sets of connected events that form the plot of stories. The connections are the causal links which give coherence to the plot. In narrative theory, this interface could be said to explore the story's fabula. Allen et al. [5] developed an interface for presenting causal explanations of scientific models. Specifically, that work described a geological hypothesis as a set of state changes. Events are central for most of these interfaces and events have been of broad general interest (e.g., $[6,7,8])$. 
In a different tradition, it is also worth noting hypertext guided tours (e.g., [9, 10]) which provide a linear framework for presentation of texts. These do not claim any particular connection among the pages, but it seems likely that the connections would often be used to connect a causal or narrative thread.

The current project explores the combination of narrative and causal links with timelines. Specifically, we present causal threads which connect coherent sets of events. Such interfaces should be helpful for supporting navigation through explanations of historic events as well as comprehension of and memory for the relationships of those events. Thus, these interfaces could improve history education.

\section{Implementation}

\subsection{Content}

As sample content, we used an analysis of the causes of the Civil War. ${ }^{1}$ This analysis proposed five threads which lead to Secession of the South from the Union. We slightly refocused some of the threads to emphasize narrative relationships among the events. Specifically, we used: Cultural/Conflict, States Rights, Abolitionists, Slavery in the New Territories, Abolitionists., and Lincoln as the five Causes of the Civil War.

In some cases, the events which composed each of these threads were simple causal chains. For instance, the development of the Cotton Gin directly changed agriculture in a way that increased the number of slaves. However, the events in some of the other threads suggested more of a trend than a simple causal chain. Thus, while we can say that Abolitionism led to the book Uncle Tom's Cabin, the effects of that book, although powerful, were too diffuse to easily represent with the current model.

\subsection{Interface}

We have developed a prototype interface for presenting causal links and threads as a stand-alone Java application. The main screen of the interface is a focus-context timeline framework related to [5]. However, the focus view is subdivided into separate threads. Initially, the threads are in a compressed or closed format (Fig. 1). Each of them can be opened by clicking on its label. Fig 2, shows the full expansion of all five threads.

As noted above, the threads are like narratives or guided tours. They generally provide a coherent presentation through the sequence of events. In our interface, each thread may be activated with the "Play thread" button which is below the thread label. When that button is selected, the "Play thread" button is replaced with "forward" and "back" arrows and the thread title is highlighted. The events in the active thread are highlighted one-by-one and a text box about it is presented.

\footnotetext{
${ }^{1}$ http://americanhistory.about.com/od/civilwarmenu/a/cause_civil_war.htm
} 


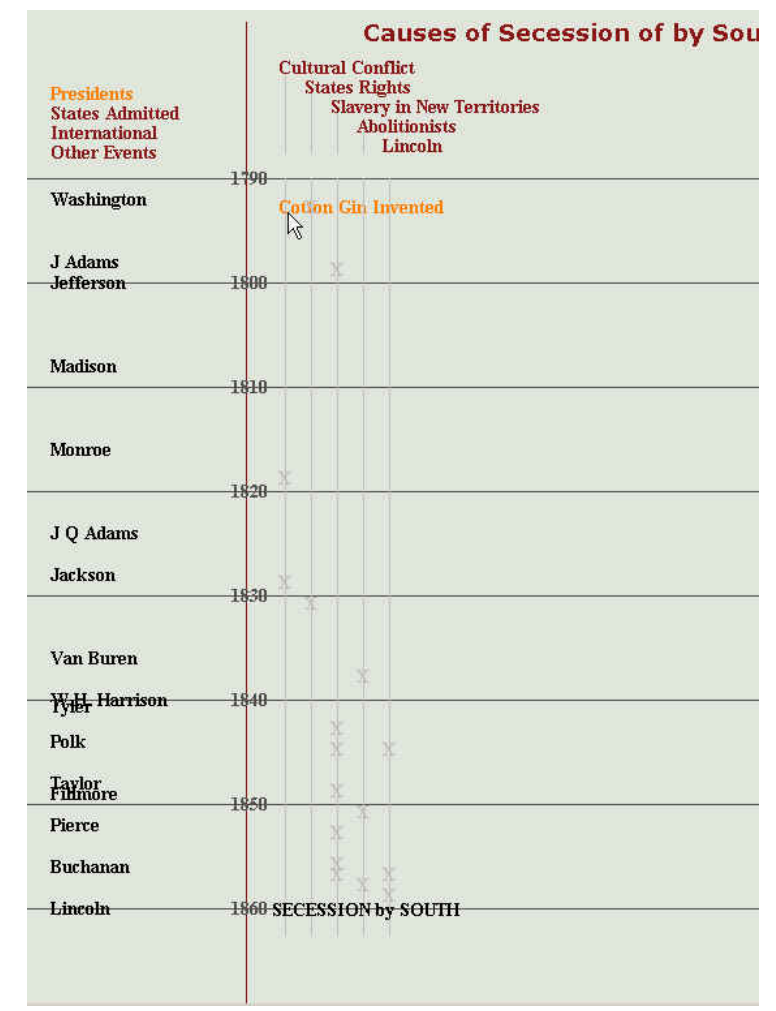

Fig. 1: A portion of the narrative timeline page I shown. On the left, a context timeline shows presidents. Other options are also able to be selected. The historical threads are shown on the right in the closed configuration. The events for each thread are indicated with an " $X$ ". Hovering over the "X" pops up a tooltip-like label for the event. Thus, the user can quickly scan a set of events. In addition, each of the historical threads can be opened by clicking on the label associated with it.

Clicking on the event label when a thread is being played produces a short menu which lists other resources associated with that event. In the current implementation, there are up to three resources: links to a sub-thread, links to Wikipedia, and the audio description. The sub-threads are focused timelines which present finer-grained subevents. For instance, the "Amistad" sub-thread elaborates the mutiny, trial, and convictions. The links to Wikipedia provide an even richer resource for background such as biographies of people and documentation. 


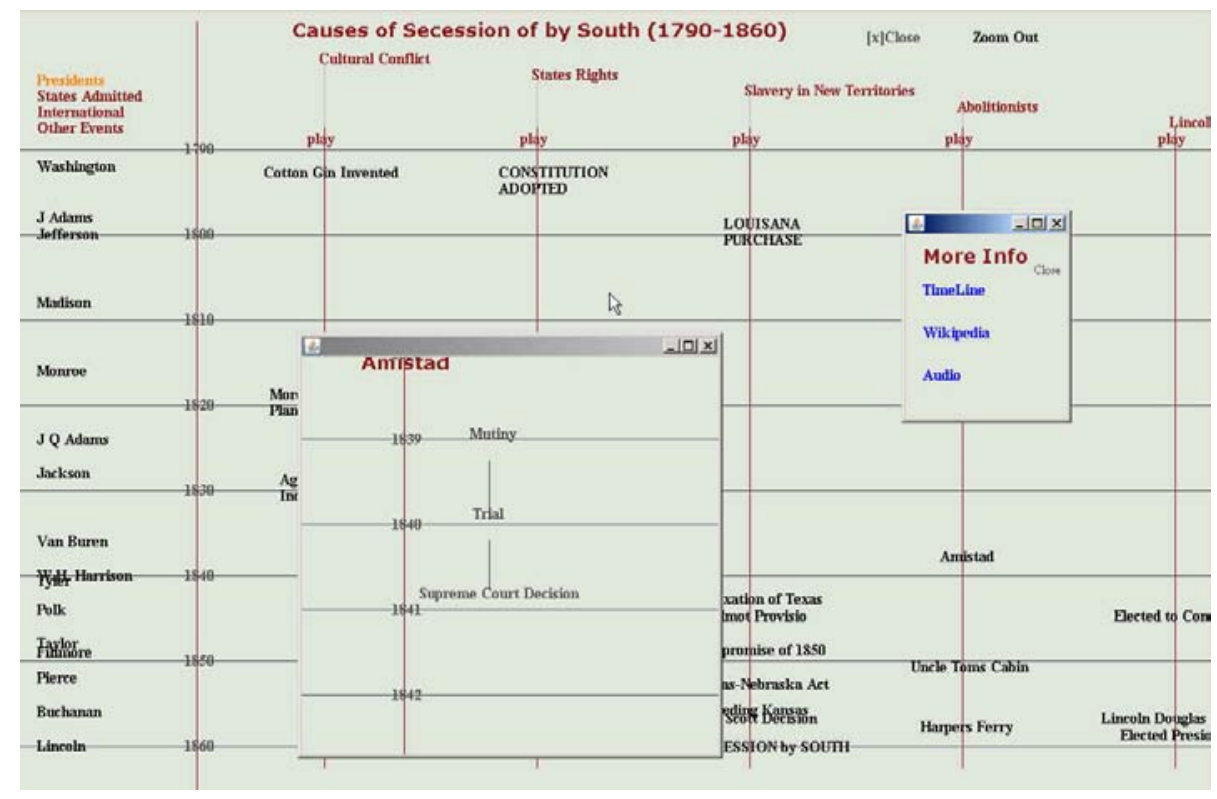

Fig. 2: In the open configuration, each of the threads has been expanded with full labels presented. A menu indicating links for additional information for the "Amistad" has been opened. Furthermore, a timeline with the detailed timeline has been opened.

We have focused thus far on the thread interface. However, we envision a broader system composed of many such thread interfaces. As a step in that direction, we developed a home page (Fig. 3) which displayed links to various sets of narrative timelines. (In the current implementation all but the "Causes of the Civil War" were stubs). Originally, we had developed a text-only outline for the home page, but many of the threads did not fit the hierarchical outline structure. The graphical approach in the figure allows multiple overlapping threads.

\section{Conclusion}

We have developed a prototype interface for incorporating narrative and causal threads in an interactive focus-context, interactive timeline environment. Moreover, each of the threads can be "played". That is, the user can step through the events in the thread. 


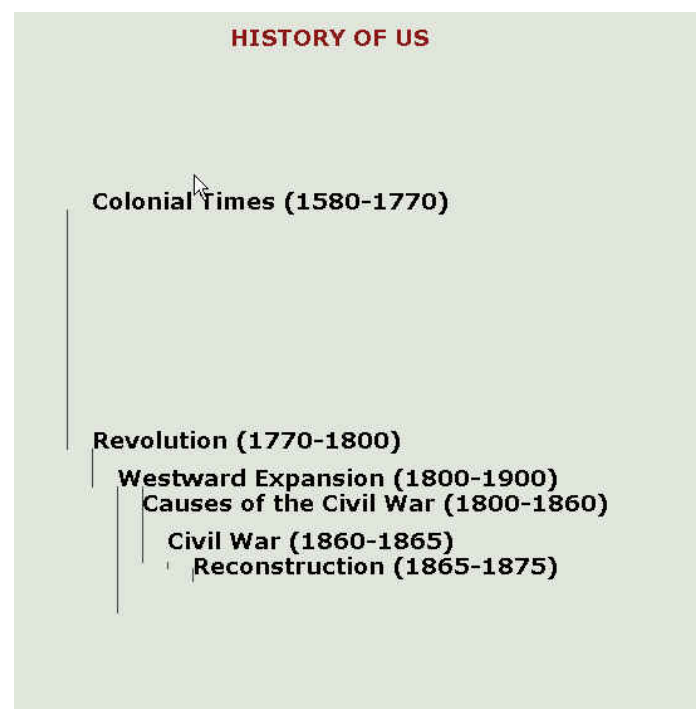

Fig. 3. This approach can be applied to major episodes of US history. The history browser home page is shown. At present only the "Causes of the Civil War" link is functional, as described above.

\section{Extensions}

Several useful features could be added to this system. For instance, we could incorporate aspects of adaptive hypertexts (e.g., [11]). For instance, when playing multiple threads, the system could adapt the node descriptions based on what other nodes had previously been presented to that same user. We might also allow individuals to add commentary and even arrange their own versions of the causal associations of links.

However, there are also several unresolved issue with the design which may affect its generality. First, some historical reasoning is based on trends and these are not easy to represent as events. For instance, the impact of the Abolitionist movement would have been based more on public acceptance of its arguments than in the direct effect of the events associated with it. A second limitation is that many event threads are not easily linearized. Rather, they are probably best represented as tangled webs,

Moreover, some of the events get deeper and could be expanded into their own full thread. For instance, the "Cotton Gin" node could be expanded into an elaborate story of how the cotton gin was developed. Similarly, "Secession of the South" could be followed by the whole story of the attack of Fort Sumter and a large network of links dealing with the Civil War. This is amplified by the inclusion of external resources. Though we could also invert the viewpoint and consider the multi-timeline system we have developed to be a useful adjunct for providing structure to those external resources. Alternatively, these threads could be used as a way to create guided tours through Wikipedia. 
Presumably, many of these issues can be mitigated. We are currently developing formalisms for describing events and the relationship among them which extends the model in [5]. Such a framework should facilitate developing larger and richer applications.

\subsection{Applications}

Large scale applications should help history students understand both temporal and causal relationships in history. Such a system could also support access to the millions of pages of historical newspapers which have are currently being digitized $[12,13,14]$. However, to apply these interfaces to such a large number of newspapers would entail the automatic extraction categorization of events from the text and such techniques still need to be developed.

Acknowledgements: This work was funded by an NEH Digital Humanities Start-up Grant. We thank Abhijeet Ganachari for assistance.

\section{References}

1. Allen, R.B., Timelines as Information System Interfaces. In: Proceedings International Symposium on Digital Libraries. pp. 175-180 (1995)

2. Allen, R.B., A Focus-Context Timeline Interface for Browsing Historical Newspapers. In: Proceedings Joint ACM-IEEE Digital Libraries Conference. pp. 260-261 (2005)

3. Plaisant, C., Milash, B., Rose, A., Widoff, S., Shneiderman, B. Life Lines: Visualizing Personal Histories. In :ACM SIGCHI . 221-227, (1996)

4. Allen, R.B., Acheson, J.A., Browsing Structured Multimedia Stories. In Proceedings ACM Digital Libraries Conference. pp. 11-18 (2000)

5. Allen, R.B., Wu, Y.J., Luo, J., Interactive Causal Schematics for Scientific Explanations. In: International Conference on Asian Digital Libraries. pp. 411-415 (2005)

6. Allen, R.B., Developing a Query Interface for an Event Gazetteer. In: Proceedings Joint Digital Libraries Conference. pp. $72-73$ (2004)

7. Shaw, R., Larson, R.R., Event Representation in Temporal and Geographic Context. In: European Conference on Digital Libraries. pp. 415-418 (2008)

8. Pustejovsky, J., Castaño, J., Ingria, R., Saurí, R., Gaizauskas, R., Setzer, A., Katz, G. TimeML: Robust Specification of Event and Temporal Expressions in Text. IWCS-5, Fifth International Workshop on Computational Semantics. (2003)

9. Marshall, C., Guided Tours and On-line Presentations: How Authors Make Existing Hypertext Intelligible for Readers. In: ACM Hypertext (1989)

10. Zellweger, P.T., Scripted Documents: A Hypermedia Path Mechanism. In: ACM Hypertext (1989)

11. Hitzeman, J, Mellish, C, Oberlander, J., Dynamic Generation of Museum Web Pages: The Intelligent Labelling Explorer, Archives and Museum Informatics, 11, 107-115 (1997)

12. Allen, R.B., Japzon, A., Achananuparp,P., Lee, K-J., A Framework for Text Processing and Supporting Access to Collections of Digitized Historical Newspapers. HCI International Conference. (2007). 
13. Allen, R.B., Waldstein, I., Zhu, W., Automated Processing of Digitized Historical Newspapers: Identification of Segments and Genres. In: International Conference on Asian Digital Libraries. pp 380-387 (2008)

14. Allen, R.B., Hall, C., Zhu, W., Automated Processing of Digitized Historical Newspapers beyond the Article Level: Finding Regular Features, Sections, and Genres of Images, (submitted) 